\title{
Influence in cladding mode distribution of overlay deposition on long-period fiber gratings
}

\author{
Ignacio Del Villar, Ignacio R. Matias and Francisco J. Arregui \\ Departamento de Ingeniería Eléctrica y Electrónica, Universidad Pública de Navarra, 31006 \\ Pamplona, Spain. \\ ignacio.delvillar@unavarra.es, natxo@unavarra.es, parregui@unavarra.es
}

\begin{abstract}
A thin overlay of higher refractive index than the cladding of a long-period fiber grating (LPFG) induces in cladding modes strong variations in effective index, mode profile, cross-coupling coefficient with the core mode, and self-coupling coefficient. Some conditions must be met in order to obtain the highest induction. The key parameters are the thickness and the refractive index of the overlay, and the ambient refractive index. Under optimum conditions, the sensitivity of the device to variations in any of the critical parameters is improved in a great manner. The result is large shifts of the attenuation bands in the transmission spectrum. If the refractive index of the overlay is complex there is an additional phenomenon of vanishing of the attenuation bands in the transmission spectrum. This occurs for specific thickness values of the overlay. The problem is solved in two steps: a vectorial analysis of the modes and the application of coupled mode theory.
\end{abstract}

OCIS codes: $050.2770,060.2430,310.1860$.

\section{INTRODUCTION}


Long Period Fiber Gratings (LPFGs) consist of index modulation of the refractive index of the core of a single mode fiber (SMF), with a much longer period than Fiber Bragg Gratings (FBGs). They play an important role mainly in two fields. Firstly, in optical communication, many devices have been developed, such as gain equalizers, ${ }^{1}$ band rejection filters, ${ }^{2}$ tunable filters ${ }^{3}$ and optical switches. ${ }^{4,5}$ Sensors are the second field. ${ }^{6-8}$ If compared with FBGs, modes couple in a different way, which improves the characteristics of sensors in a great manner. There is a coupling between the core and the copropagating cladding modes, which makes the device sensitive to the surrounding media. This also includes the drawback of a dependence on temperature, but there exist techniques for avoiding this problem, ${ }^{7}$ which permits multiparameter sensing. ${ }^{8}$

The fabrication of LPFGs can be obtained with several techniques, being ultraviolet (UV) irradiation the most extended one. Others are ion implantation, irradiation by femtosecond pulses in the infrared, irradiation by $\mathrm{CO}_{2}$ lasers, diffusion of dopants into the core, relaxation of mechanical stress, and electrical discharges. A good review on these techniques can be found in Ref. 9.

Regarding numerical methods for analysis of LPFGs, two different cases have been studied so far. The first case includes LPFGs surrounded by an infinite medium refractive index lower than the cladding. In Ref. 10 there is a study with hybrid modes and a uniform azimutal perturbation, whereas in Ref. 11 the LP mode approximation is used for arbitrary azimutal perturbation. As the ambient refractive index approaches that of the cladding, the sensitivity of the resonance wavelength to variations of the ambient refractive index is higher. Then, the second case starts when the ambient refractive index exceeds that of the cladding. The core mode couples with radiation modes, ${ }^{12-14}$ and the dependence of the resonance wavelength on the ambient refractive 
index is not so straightforward. However, the resonance depth is more dependent on this parameter for values close to the refractive index of the cladding. ${ }^{6}$ In both cases, the region of highest sensitivity is located around the refractive index of the cladding.

Recently, a third case has been analysed both theoretically and experimentally. ${ }^{15-17}$ In the previous two cases, the cladding was surrounded by a medium of infinite thickness. Now a thin overlay of higher refractive index than the cladding is deposited between the cladding and the surrounding media. One of the cladding modes will be guided by the overlay if this is thick enough. ${ }^{16}$ This causes a reorganization of the effective indices of the modes of the cladding. As a result, there are important variations of the Bragg condition, which leads to wide shifts of the resonance wavelengths if we work around the thickness value where there is a transition to guidance of a cladding mode in the overlay. The aim is to select, for a specific ambient refractive index, an adequate refractive index and thickness of the overlay. In this way, it is increased the sensitivity of the effective index of the cladding modes for a specific application. Once an optimum design has been obtained, the variation of either the refractive index of the overlay or the ambient, or the thickness of the overlay, leads to much higher resonance wavelength shifts than in traditional LPFGs without the presence of an overlay on the cladding. The applications of this include higher sensitive LPFG devices, optical filters and optical switches.

In addition to this, there is a second issue to keep in mind. So far, only Electrostatic SelfAssembly (ESA) ${ }^{17}$ and Langmuir Blodgett ${ }^{15}$ techniques have been applied for the deposition of uniform coatings with thicknesses of tens of nanometers around the cylindrical shaped substrate. In both cases the attenuation bands vanish for a range of thickness values and reappear as the thickness of the overlay increases. It was proved in Ref. 18 that some ESA materials can present a high imaginary part due to scattering and material losses. If the imaginary part of the material 
of the overlay is considered, theoretical and experimental results agree. Anyway other factors may contribute to the phenomenon, such as insertion losses in the transition of fiber with overlay and fiber without overlay, radiation, and the variation of the cross-coupling coefficient. This last factor has no influence with the LP mode approximation, ${ }^{16}$ whereas it plays an important role with the hybrid mode analysis presented in this work. The vanishing of the attenuation bands coincides with the guidance of one of the cladding modes in the overlay as it will be explained in section 3. The consequence of this vanishing of modes is negative in terms of wavelength shift detection, because it limits the overall shift of the attenuation bands. This can be avoided by paying attention to other hybrid modes whose attenuation bands appear when the rest of attenuation bands vanish. On the other hand, high variations in amplitude can be exploited in the same manner as wavelength shift in sensors applications. Furthermore, the theory presented can be applied to other structures with nanodeposition on a substrate. ${ }^{19}$

In Refs.16 and 17 a numerical method was used for determining the wavelength that satisfies the Bragg condition between the core mode and each cladding mode, and the transmittance of the LPFG is based on that described in Ref. 11. Coupled mode theory was the basis for the calculation of LP modes in a cylindrical multilayer waveguide. In this work, the more exact formulation for hybrid modes used for the three layer mode ${ }^{10}$ is applied for the four layer model presented in section 2. The LPFG is analyzed in two steps. First, in section 2 there is a study of all parameters involved in the transversal section of the LPFG. The distribution of modes as a function of the overlay is analyzed in depth. Second, in section 3 the longitudinal problem is solved with the coupled mode theory, which is applied for the calculation of the transmission spectra in LPFGs. Finally, some conclusions are given in section 4.

\section{MODE ANALYSIS IN A FOUR-LAYER CYLINDRICAL WAVEGUIDE}


In Fig. 1 an LPFG structure is represented with an overlay deposited on the cladding. Its transversal section is a four-layer cylindrical structure: core, cladding, overlay and ambient. Coupled mode theory has been applied for the simulation of LPFG structures without an overlay. In Ref. 11 a three-layer model based on the LP mode approximation is presented, whereas in Ref. 10 the more exact hybrid modes are calculated. In the last case it was considered for the sake of simplicity that there was no azimutal variation of the perturbed index profile after exposure to UV radiation. In this way, there are only interactions between the core mode $H E_{1, l}$, and $H E_{l, j}$ and $E H_{l, j}$ modes of the cladding. Both methods led to similar results because $L P_{0, j}$ modes were very similar to $H E_{1, j}$ modes, and because $E H_{1, j}$ modes that were not considered in the LP mode approximation had negligible cross-coupling coefficients with the core mode $\left(H E_{l, 1}\right)$ to play a role in the transmission spectrum.

It has been recently proved with the LP mode approximation that cladding modes in an LPFG can be perturbed with the presence of an overlay of higher refractive index than the cladding. ${ }^{16}$ The theory used to analyze the four-layer problem was the same as that of the three-layer problem. Now we prove that the LP mode approximation is not always valid for calculating $H E_{l, j}$ modes. If the hybrid modes are calculated for the four-layer model some differences can be extracted.

To analyze this fact, the same optical fiber of Ref. 10 and 11 is selected. The core diameter is 5 $\mu \mathrm{m}$, the cladding diameter $125 \mu \mathrm{m}$, core refractive index 1.4573, cladding refractive index 1.45 , The parameters of the long-period gratings are: period and amplitude of the sinusoidal modulation of the core refractive index: $276 \mu \mathrm{m}$ and $2.7 \times 10^{-4}$ respectively, length of the grating $25 \mathrm{~mm}$, and the modulation is considered sinusoidal, consequently $\sigma(\mathrm{z})=\mathrm{s}_{0}=\mathrm{s}_{1}=1$. The material for the overlay is $\left[\mathrm{PDDA}^{+} / \mathrm{PolyR}-478^{-}\right]$with an approximate refractive index of $1.62 .{ }^{17}$ 
This value was considered constant in the wavelength range selected. The notation used for the modes is: $\mathrm{HE}_{1,1}$ for the core mode; $\mathrm{HE}_{1,2}$ for the first $\mathrm{HE}_{1, \mathrm{j}}$ cladding mode, $\mathrm{HE}_{1,4}$ for the second $\mathrm{HE}_{1, \mathrm{j}}$ cladding mode, and so on; $\mathrm{EH}_{1,3}$ for the first $\mathrm{EH}_{1, \mathrm{j}}$ cladding mode, $\mathrm{EH}_{1,5}$ for the second $\mathrm{EH}_{1, \mathrm{j}}$ cladding mode, and so on.

If an overlay of higher refractive index than the cladding is deposited on an LPFG, as the thickness of the overlay increases, there is a moment when the overlay starts guiding a mode. It is exactly the cladding mode with highest effective index that jumps to the overlay. If the thickness continues to be increased, more modes are guided in the overlay. The mode that jumps to the overlay is always the highest effective index mode of the group of cladding modes that are not guided in the overlay. ${ }^{16}$ In the transition to guidance of each of these modes, a fast change in the effective index of each cladding mode occurs. After this transition takes place, the effective index of each cladding mode is that of the corresponding lower one before the transition.

In Fig. 2 the effective index of the core mode and the first fifteen cladding modes are represented as a function of the overlay thickness for a fixed wavelength of $1200 \mathrm{~nm}$. The $\mathrm{HE}_{1,2}, \mathrm{HE}_{1,4}$, $\mathrm{HE}_{1,6}$, modes become guided in the overlay at about 260, 1090 and $1920 \mathrm{~nm}$ (see Fig. 2b), which coincides with $\mathrm{LP}_{0,2}, \mathrm{LP}_{0,3}, \mathrm{LP}_{0,4}$ modes. The guidance in the overlay is manifested by the sudden effective index change at these overlay thickness values. As the overlay thickness increases, the effective index of each of these modes trends to the overlay refractive index, which indicates that the modes are more confined in the overlay region. Around the transition to guidance of each of the modes in the overlay, the higher cladding modes cover the energy state left by their predecessors. For instance, at $260 \mathrm{~nm}$ the effective index of the $\mathrm{EH}_{1,3}$ mode will be now that of the $\mathrm{HE}_{1,2}$ mode, the effective index of the $\mathrm{HE}_{1,4}$ mode will be that of the $\mathrm{EH}_{1,3}$, the effective index of the $\mathrm{EH}_{1,5}$ mode will be that of the $\mathrm{HE}_{1,4}$, and so forth. The main difference in comparison with 
LP mode approximation is the presence of $\mathrm{EH}_{1, \mathrm{j}}$ modes. Instead of the unique guidance of an $\mathrm{LP}$ mode, now there is a guidance of a pair of modes: one HE and one EH mode. Since they are guided in a short thickness interval, it seems that the higher order HE modes jump to their preceding HE mode, and that the same is true for EH modes. Truly there is a two step transition. This fact is more easily appreciated for low-order cladding modes (see $\mathrm{HE}_{1,8}$ cladding mode in Fig. 2a), because transitions take place in a shorter thickness interval. For higher order modes it seems that there is a one step transition, as it is the case in Fig. 2a for the $\mathrm{HE}_{1,16}$ cladding mode. Moreover, it can be concluded that $L P_{0, j}$ cladding modes match $H E_{1, j}$ cladding modes no matter the thickness of the overlay when its effective index is sufficiently different to that of the core mode. This is confirmed in section 3 by analyzing the resonance wavelengths in the transmission spectrum of the LPFG as a function of the overlay thickness. On the other hand, as the refractive index contrast between the cladding and the overlay decreases, the weak guidance condition is met. Consequently, both LP and hybrid mode formulations coincide in all cases.

The phenomenon of Fig. 2a can be understood in terms of reorganization of modes. There exist allowed states for the effective indices of the modes when there is not a thin overlay around the LPFG. When the structure is perturbed by the deposition of a thin overlay compared to the thickness of the structure, there are not-allowed states that coincide with the transition to guidance of one cladding mode (the mode of highest energy) in the overlay. This occurs when a specific thickness value of the thin overlay is reached. As the thickness increases, the effective index distribution of the modes recovers its original aspect. The phenomenon repeats more times for periodically spaced thickness values of the overlay.

The phenomenon is also confirmed by analyzing the fields of cladding modes in Fig. 3. The $\mathrm{HE}_{1,4}$ cladding mode has been selected. It presents a two step transition at about $300 \mathrm{~nm}$ (see Fig. 
2a). In this way, the field profile of the mode must transit firstly to the $\mathrm{EH}_{1,3}$ cladding mode, and secondly to the $\mathrm{HE}_{1,2}$ mode. For the sake of comparison, the radial electric field of $\mathrm{HE}_{1,4}, \mathrm{EH}_{1,3}$ and $\mathrm{HE}_{1,2}$ modes has been also represented in Fig. 3 for the LPFG structure without deposition. For an overlay of $100 \mathrm{~nm}$ the mode distribution is still stable, consequently $\mathrm{HE}_{1,4}$ mode profile resembles that of the structure without deposition. For $255 \mathrm{~nm}$, the $\mathrm{HE}_{1,4}$ mode is mutating to the profile of the $\mathrm{EH}_{1,3}$ mode without deposition. Note that at $300 \mathrm{~nm} \mathrm{HE} \mathrm{H}_{1,4}$ mode is similar to $\mathrm{EH}_{1,3}$ mode without overlay; the transition to an $\mathrm{EH}$ mode is completed. At $345 \mathrm{~nm}$ the $\mathrm{HE}_{1,4}$ mode is following the second transition to the $\mathrm{HE}_{1,2}$ mode of the structure without deposition. Finally at $500 \mathrm{~nm}$ it can be asserted that the mode profile $\mathrm{HE}_{1,4}$ is the same as that of the $\mathrm{HE}_{1,2}$ mode of the structure without overlay.

Finally, an additional contribution to the confirmation of the mutation of modes is the analysis of the self-coupling coefficients. If we represent in Fig. 4 the self-coupling coefficient of the $\mathrm{HE}_{1,6}$ mode as a function of the overlay thickness we can see that when it mutates to the $\mathrm{EH}_{1,5}$ mode of the structure without overlay, its coupling coefficient trends to the original coupling coefficient of the $\mathrm{EH}_{1,5}$ mode at about $250 \mathrm{~nm}$. Later on it trends to the original $\mathrm{HE}_{1,4}$ coupling coefficient at about $350 \mathrm{~nm}$. The same occurs at $1100-1200 \mathrm{~nm}$. Its coupling coefficient becomes that of $\mathrm{EH}_{1,3}$ mode in a first step at about $1100 \mathrm{~nm}$ and that of $\mathrm{HE}_{1,2}$ in a second step at $1200 \mathrm{~nm}$. The explanation is also valid for the evolution of $\mathrm{EH}_{1,7}$ mode. The same phenomenon occurs with the cross-coupling coefficients, as it will be proved later.

To finish this section, the possibility that the overlay presents losses is considered. This problem is introduced by analyzing the effect in the effective index of cladding modes, which helps to understand some of the spectra obtained in section 3. It has been proved with ESA method that the nanodeposition of a thin film at the end of a multimode fiber causes important losses. ${ }^{18}$ The 
causes are the inherent losses of the material and scattering losses due to roughness. Considering these factors as an imaginary part of the refractive index of the material deposited, permits that results agree with theory. For the example presented here it will be assumed that the material has a refractive index of $1.62+0.0025 \mathrm{i}$. In this case, the pair of cladding modes that are guided at the first two-step transition are $\mathrm{HE}_{1,2}$ and $\mathrm{EH}_{1,3}$, which are the highest states of energy. However, this is not always the case, and this rule may be broken if the imaginary part of the refractive index is high enough. In some case it is not the highest state of energy that is guided in the overlay. The interpretation of this phenomenon is beyond the scope of this work, but if the imaginary part of the effective index of cladding modes is represented as a function of the overlay thickness (see Fig. 5) this offers a good reference for the transmission spectra of LPFGs with lossy overlays. The first eight $\mathrm{HE}_{1, \mathrm{j}}$ modes have been plotted. At the transition to guidance in the overlay of the $\mathrm{HE}_{1,2}$ and $\mathrm{EH}_{1,3}$ cladding modes $(260$ and $350 \mathrm{~nm}$ ) the imaginary part in all modes reaches a maximum. But the maxima are different depending on the mode order. For higher order modes, the second maximum is very low compared to the first one, whereas for lower order modes the second maximum is comparable or even higher than the first one. It was proved in Fig. 2 that for higher order modes there is a one step transition. As a result, it can be concluded that the influence of one of the two guided modes in the overlay has a negligible effect in the effective index evolution of the cladding modes. In this way, it can be understood why the second maximum for higher order modes is so low. Similarly, for higher EH modes the first maximum is very low if compared with the second one.

\section{TRANSMISSION SPECTRUM ANALYSIS IN LPFG STRUCTURES WITH AN OVERLAY ON THE CLADDING}


After obtaining the modes in the four-layer cylindrical waveguide, and obtaining the self- and cross-coupling coefficients in the transversal section, the coupled-mode differential equations are applied for solving the longitudinal problem. ${ }^{10,11,16}$ This permits to obtain the transmission spectrum where clear attenuation bands are appreciated. They are produced by coupling between the core mode and the copropagating cladding modes.

When the goal is to see the displacement of the resonance wavelengths, alternative solutions to the application of the differential equations can be used.

The first one is the calculation of the resonance wavelength with the Bragg condition:

$$
\beta_{11}(\lambda)-\beta_{1 j}(\lambda)=\frac{2 \pi}{\Lambda}
$$

where $\beta_{l l}$ and $\beta_{l j}$ are the propagation constants of the core and the $j$ cladding modes respectively, and $\Lambda$ is the period of the grating. Results obtained present appreciable variations related to those values calculated with coupled-mode differential equations. However, if the modified first-order Bragg condition is applied, errors are lower than $0.1 \%^{11}$ :

$$
\beta_{11}(\lambda)+s_{0} \zeta_{11,11}(\lambda)-\left(\beta_{0 j}(\lambda)+s_{0} \zeta_{1 j, 1 j}(\lambda)\right)=\frac{2 \pi}{\Lambda}
$$

where $\varsigma_{01,01}$ and $\varsigma_{0 j, 0 j}$ are the self-coupling coefficients of the core and the $j$ cladding modes, and $\mathrm{s}_{0}$ is the coefficient of the first Fourier component of the grating function $\mathrm{S}(\mathrm{z})$. If this error is compared with fabrication tolerances, it can be concluded that this approximation offers great advantages in terms of computational effort. Henceforward it will be used in some cases in next section.

The reorganization of the effective indices of the modes of the cladding observed in section 2 has important consequences in the resonance wavelengths of the transmission spectra of LPFGs. There are important variations of the Bragg condition, which leads to dramatic shifts of the 
resonance wavelengths if it is being worked around the thickness value where the cladding mode starts being guided in the overlay. The LP mode approximation, though not exact, permitted to understand the phenomenon and results did not differ greatly from those obtained with a method that considers both HE and EH modes. LP mode approximation is the basis for understanding the differences due to the utilization of a more exact method. The main novelty is that for each LP cladding mode, one HE and one EH cladding modes are guided in the overlay. Since the EH modes were not considered in the LP mode approximation its influence in the transmission spectrum must be considered. In addition to this, if the overlay presents important losses the guided mode is not always the one that presents a higher effective index (highest energy state) and there is an additional phenomenon of vanishing of the resonance. For the sake of simplicity, this section is divided into two different parts: deposition of overlays without losses and deposition of overlays with losses.

\section{A. Deposition of overlays without losses}

The immediate consequence of the shift in effective index is that it leads to a displacement in all the attenuation bands. With the LP mode approximation the conclusion is that there is an optimal deposition thickness where the central wavelength shift as a function of the ambient refractive index is highest. ${ }^{16}$ This is the optimum overlay thickness (OOT). This value depends mainly on three variables: the refractive index of the overlay and the ambient refractive index, and the overlay thickness. However, because of the two step transition there are now two OOTs. To calculate the OOTs, either the modified Bragg condition or the couple mode equations explained in section 2 will be used. The derivative of the wavelength with respect to the overlay thickness will define the optima. 
The modified Bragg condition is more effective in terms of computation than the coupled mode equations. So, it is used for calculating the resonance wavelength shifts as a function of the overlay thickness. In Fig. 6 it is shown the evolution of the resonance wavelength caused by coupling between the first fifteen cladding modes and the core mode. In comparison with LP mode approximation, there is a contribution of $\mathrm{EH}_{1, \mathrm{j}}$ modes. Like in Fig. 2a, there is a two step transition at about $300 \mathrm{~nm}$ of higher order resonances to lower order ones. Firstly the $\mathrm{HE}_{1,2}$ mode is guided in the overlay. The consequence is that the $\mathrm{EH}_{1,3}$ mode resonance wavelength shifts to that of the $\mathrm{HE}_{1,2}$ mode when no overlay is present. The same is true for the $\mathrm{HE}_{1,4}$ mode, which shifts its resonance wavelength to that of the $\mathrm{EH}_{1,3}$. The same occurs for the rest of higher effective index mode resonances.

Secondly, $\mathrm{EH}_{1,3}$ mode is guided in the overlay. The $\mathrm{HE}_{1,4}$ mode resonance wavelength shifts to that of the $\mathrm{EH}_{1,3}$ mode before deposition started. The same is true for the $\mathrm{EH}_{1,5}$ mode, which shifts its resonance wavelength to that of the $\mathrm{HE}_{1,4}$. The same occurs for the rest of higher effective index mode resonances.

For low-order cladding mode resonances, the two step transition of $\mathrm{HE}_{1, \mathrm{j}}$ modes resonances to their immediate lower order $\mathrm{HE}_{1, \mathrm{j}}$ modes resonances can be clearly appreciated. The same is true for $\mathrm{EH}_{1, \mathrm{j}}$ mode resonances. For higher order modes it seems that there is a one step transition. This could be expected by taking a look at Fig. 2a, where the two step transition is clear for loworder cladding modes (i.e. the $\mathrm{HE}_{1,8}$ mode), and not for higher order cladding modes (i.e. the $\mathrm{HE}_{1,16}$ mode). Consequently, the LP mode approximation remains correct for higher order $\mathrm{HE}_{1, \mathrm{j}}$ mode resonances. This is corroborated by comparing in Fig. 7 the derivative of the resonance wavelength with respect to the overlay thickness for both LP and hybrid mode approximations. However, it is important to note that even though it seems that there is a one step transition for 
higher order mode resonances, the second OOT still remains visible (see $\mathrm{HE}_{1,16}$ mode in Fig. 7). As explained in section 2, the agreement between both formulations will be reached for all modes at the weak guidance condition (low refractive index contrast between the cladding and the overlay). As an example, in Fig. 8 it is compared the $\mathrm{HE}_{1,16}$ mode resonance wavelength shift as a function of the overlay thickness for two different overlay refractive indices. It is clear that the weak guidance condition is met for a refractive index 1.55 (low contrast with the cladding), whereas for 1.8 (high contrast with the cladding) the two transitions are very clear. Another important point is that the OOT varies as a function of the overlay refractive index. This indicates that the same evolution of the resonance wavelengths is produced if the overlay thickness and ambient refractive index are fixed, and the overlay refractive index varies. The same effect is expected with the ambient refractive index, which is the third key parameter for the definition of the OOTs.

If it is desired to analyze the depth of the attenuation bands in the transmission spectrum, the coupled mode equations must be used. With the LP mode approximation it was checked that no vanishing of the attenuation bands was appreciated when the refractive index of the overlay is purely real. ${ }^{16}$ However with the formulation of this chapter it is checked that the presence of $\mathrm{EH}_{1, \mathrm{j}}$ modes plays an important role. After the highest cladding mode is guided by the overlay and before the second cladding mode is guided, the $\mathrm{EH}_{1, \mathrm{j}}$ mode resonances are appreciable in the transmission spectrum, and $\mathrm{HE}_{1, \mathrm{j}}$ mode resonances diminish because its field profile resembles to that of a $\mathrm{EH}_{1, \mathrm{j}}$ mode. In Fig. 9 it is shown the spectrum of the $\mathrm{HE}_{1,16}$ and $\mathrm{HE}_{1,14}$ mode resonances for five different overlay thickness values: 0, 255, 289 and $335 \mathrm{~nm}$. Note that at $750 \mathrm{~nm}$ the transmission spectrum has recovered its original aspect before deposition and superposes the spectrum obtained for without overlay. For this reason we have not included it in Fig. 9. $\mathrm{EH}_{1,15}$ 
mode resonance is clearly appreciated at 289 and $335 \mathrm{~nm}$, which coincides with the second step of the transition of modes. This is caused by the increase in the cross-coupling coefficient in this thickness interval, in analogy to the analysis of the self-coupling coefficients in Fig. 4.

\section{B. Deposition of overlays with complex refractive index}

In this subsection an analysis is performed of the transmission spectra obtained when the refractive index of the overlay is complex. It is assumed like at the end of section 2 that the overlay has a $1.62+0.0025 \mathrm{i}$ refractive index.

In Fig. 10, the resonance wavelength shift for the $\mathrm{HE}_{1,16}$ mode is contrasted with the results obtained with the modified Bragg condition for an overlay without losses. It is important to note the similarity in the results, which permits to conclude that losses in the overlay do not affect the wavelength shift of the attenuation bands. Nonetheless, the depth of the attenuation is a factor affected in a great manner, oppositely to the case of an overlay with a purely real refractive index. The depth of the attenuation band as a function of the overlay thickness is also represented for the same mode. The contribution to the vanishing of the resonance at about 300 is mainly due to two factors: the imaginary part of the overlay refractive index and the intermediate transition of the HE mode to a EH mode profile, which causes a reduction in the cross-coupling coefficient. Note that the discontinuities in the results are caused by the $0.2 \mathrm{~nm}$ resolution used in this case.

To visualize the shape of the spectrum, the same four overlay thickness values of Fig. 9 are analyzed in Fig. 11: 0, 255, 289 and $335 \mathrm{~nm}$. Note that the attenuation bands are not as clear as in the case without losses. Due to the imaginary part of cladding modes there is a vanishing in the attenuation bands. The explanation can be found in the coupled mode equations, ${ }^{11}$ where the imaginary part of the cladding modes introduces an attenuation factor in all cross-coupling coefficients and in the self-coupling coefficients of cladding modes. 


\section{CONCLUSIONS}

Calculation of the hybrid modes in a multilayer waveguide and coupled mode equations permits to obtain the transmission in an LPFG with an overlay. This theory complements the simpler LP approximation $^{16}$.

It has been reaffirmed that if the overlay presents a higher refractive index than the core, it starts guiding the cladding modes with the highest effective index as well as its thickness is increased. In the transition of each of these modes to guidance in the overlay there is a modal redistribution. This has been proved in terms of effective index, field representation, and coupling coefficient analysis of the modes. The consequence is a fast shift of the attenuation bands obtained in the transmission curve. However, LP mode theory is modified because of the presence of EH modes. Instead of an LP mode transition there is one HE and one EH mode transition in a short thickness interval. This causes a two step transition. This transition is not clear for high-order cladding modes, and consequently LP mode approximation remains correct. However, for low-order cladding modes, the transition is much clearer and both theories differ in a great manner if the weak guidance condition is not met.

It is known that nanodeposition techniques such as Electrostactic Self-Assembly (ESA) method present complex refractive indices. In this case there is an additional phenomenon of vanishing of attenuation bands in the transmission spectrum.

The conclusion is that an overlay of higher refractive index than the cladding acts like an external electric field perturbing the mode distribution in the LPFG. It was concluded with the LP mode approximation that after selecting an overlay with an adequate refractive index, there is an optimum overlay thickness for each combination of ambient and overlay refractive indices. With the theory of this chapter it is proved that there are two clear OOTs for low-order cladding 
mode resonances when the weak guidance condition is not met, and only one clear for high-order cladding mode resonances. For the OOT, the sensitivity of the device to variations in the ambient refractive index, the overlay refractive index, or the overlay thickness is increased in a great manner. This could be exploited for sensor purposes or even other fields in optical communications, such as optical filters or optical switches.

\section{ACKNOLEDGEMENTS}

This work was supported by Spanish Ministerio de Ciencia y Tecnologia and FEDER Research Grants CICYT-TIC 2003-00909, Gobierno de Navarra and FPU MECD Grant. 


\section{REFERENCES}

1. J. R.Qiang and H. E. Chen, "Gain flattening fibre filters using phase shifted long period fibre grating,” Electron. Lett. 34, 1132-1133 (1998).

2. A. M. Vengsarkar, P. J. Lemaire, J. B. Judkins, V. Bathia, T. Erdogan, and J. E. Sipe, "Longperiod fiber gratings as Band Rejection Filters,” J. Lightwave Technol. 14, 58-65 (1996).

3. B. J. Eggleton, R. E. Slusher, J. B. Judkins, J. B. Stark and A. M. Vengsarkar, “All-optical switching in long period fiber gratings," Opt. Lett. 22, 883-885 (1997).

4. K. W. Chung, S. Yin, "Analysis of widely tunable long-period grating by use of an ultrathin cladding layer and higher-order cladding mode coupling," Opt. Lett. 29, 812-814 (2004).

5. Y. Jeong, H. R. Kim, S. Baek, Y. Kim, Y. W. Lee, S. D. Lee and B. Lee, "Polarizationisolated electrical modulation of an etched long-period fiber grating with an outer liquidcrystal cladding," Opt. Enginering, 42, 964-968, (2003).

6. H. J. Patrick, A. D. Kersey and F. Bucholtz, "Analysis of the response of long period fiber gratings to external index of refraction,” J. Lightwave Technol. 16, 1606-1612 (1998).

7. Y. G. Han, S. B. Lee, C. S. Kim, J. U. Kang, U. C. Paek and Y. Chung, "Simultaneous measurement of temperature and strain using dual long-period fiber gratings with controlled temperature and strain sensitivities," Opt. Exp. 11, 476-481 (2003).

8. V. Bathia, “Applications of long-period gratings to single and multi-parameter sensing," Opt. Exp. 4, 457-466 (1999).

9. S. W. James and R. P. Tatam, "Optical fibre long-period grating sensors: characteristics and application," Meas. Sci Technol. 14, R49-R61 (2003).

10. T. Erdogan, "Cladding-mode resonances in short- and long-period fiber gratings filters," J. Opt. Soc. Am. A 14, 1760-1773 (1997). 
11. E. Anemogiannis, E. N. Glytsis and T. K. Gaylord, "Transmission characteristics of longperiod fiber gratings having arbitrary azimutal/radial refractive index variation," J. Lightwave Technol. 21, 218-227 (2003).

12. D. B. Stegall and T. Erdogan, "Leaky cladding mode propagation in long-period fiber grating devices,” IEEE Photon. Technol. Lett. 11, 343-345 (1999).

13. Y. Koyamada, "Numerical analysis of core-mode to radiation-mode coupling in long-period fiber gratings," IEEE Photon. Technol. Lett. 13, 308-310 (2001).

14. Y. Jeong, B. Lee, J. Nilsson and D. J. Richardson, “A quasi-mode interpretation of radiation modes in long-period fiber gratings,” IEEE J. Quantum Elec. 39, 1135-1139 (2003)

15. N. D. Rees, S. W. James, R. P. Tatam and G. J. Ashwell, "Optical fiber long-period gratings with Langmuir-Blodgett thin-film overlays," Opt. Lett. 27, 686-688 (2002).

16. I. Del Villar, I. R. Matías, F. J. Arregui and P. Lalanne, "Opitmization of sensitivity in Long Period Fiber Gratings,” Opt. Exp. 13, 56-69 (2005).

17. I. Del Villar, M. Achaerandio, I. R. Matías and F. J. Arregui, "Deposition of an Overlay with Electrostactic Self-Assembly Method in Long Period Fiber Gratings,” Opt. Lett. 30, 720-722 (2005).

18. I. Del Villar, I. R. Matias, F. J. Arregui and R. O. Claus, "ESA based in-fiber nanocavity for hydrogen peroxide detection," IEEE Trans. Nanotech. 4, 187-193, (2005).

19. G. Stewart and B. Culshaw, "Optical waveguide modelling design for evanescent field chemical sensor,” Opt. Quant. Elec. 26, S249-S259 (1994). 


\section{Figure captions}

Fig. 1: LPFG structure with deposition of an overlay on the cladding.

Fig. 2: Effective index as a function of the overlay thickness of a) first fifteen cladding modes and b) core mode and first six cladding modes.

Fig. 3: $\mathrm{n}^{2}(\mathrm{r})$ times the radial electric field of the $\mathrm{HE}_{1,4}, \mathrm{EH}_{1,3}$ and $\mathrm{HE}_{1,2}$ modes for a structure without overlay, and $\mathrm{HE}_{1,4}$ mode for five overlay thickness values: 100, 255, 300, 345 and $500 \mathrm{~nm}$.

Fig. 4: Self-coupling coefficient for $\mathrm{HE}_{1,6}$ and $\mathrm{EH}_{1,7}$ modes as a function of the overlay thickness.

Fig. 5: Imaginary effective index as a function of the overlay thickness for $\mathrm{HE}_{1,4}, \mathrm{HE}_{1,6}, \mathrm{HE}_{1,8}, \mathrm{HE}_{1,10}$, $\mathrm{HE}_{1,12}, \mathrm{HE}_{1,14}, \mathrm{HE}_{1,16}$ and $\mathrm{HE}_{1,18}$ modes

Fig. 6: Resonance wavelength shift originated by coupling between core mode and first fifteen cladding modes, as a function of the thickness of the overlay. Overlay refractive index: 1.62. Ambient index 1.

Fig. 7: Derivative of the resonance wavelength $(\lambda)$ as a function of the overlay thickness $(t)$. Comparison of a) $\mathrm{HE}_{1,16}$ and $\mathrm{LP}_{0,9}$ modes b) $\mathrm{HE}_{1,10}$ and $\mathrm{LP}_{0,6}$ modes

Fig. 8: $\mathrm{HE}_{1,16}$ resonance wavelength as a function of the overlay thickness. Overlay refractive indices: 1.55 and 1.8. Ambient index 1.

Fig. 9: Transmission spectrum showing the $\mathrm{EH}_{1,17}, \mathrm{HE}_{1,16}, \mathrm{EH}_{1,15}$ and $\mathrm{HE}_{1,14}$ mode resonances for four overlay thickness values: 0, 255, 289 and $335 \mathrm{~nm}$. Overlay refractive index: 1.62. Ambient index 1.

Fig. 10: a) $\mathrm{HE}_{1,16}$ resonance wavelength as a function of the overlay thickness with the Modified Bragg condition and Coupled-mode differential equations. b) Coupled-mode differential equations for the maximum attenuation of $\mathrm{HE}_{1,16}$ resonance. Overlay refractive indices analyzed: 1.62 and $1.62+0.0025 \mathrm{i}$. Ambient index 1.

Fig. 11: Transmission spectrum showing the $\mathrm{EH}_{1,17}, \mathrm{HE}_{1,16}, \mathrm{EH}_{1,15}$ and $\mathrm{HE}_{1,14}$ mode resonances for four overlay thickness values: $0,255,289$ and $335 \mathrm{~nm}$. Overlay refractive index: 1.62+0.0025i. Ambient index 1 . 
Fig. 1

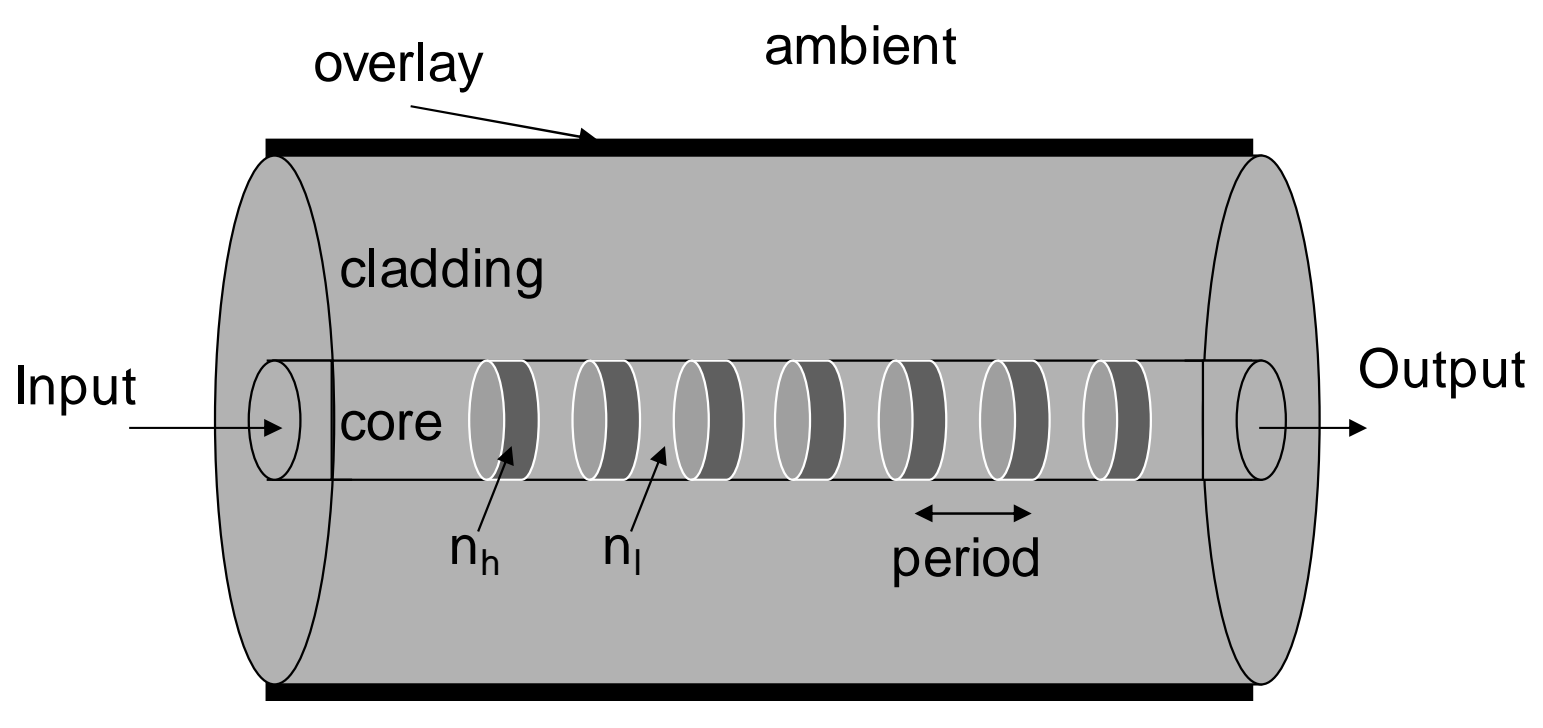


Fig. 2
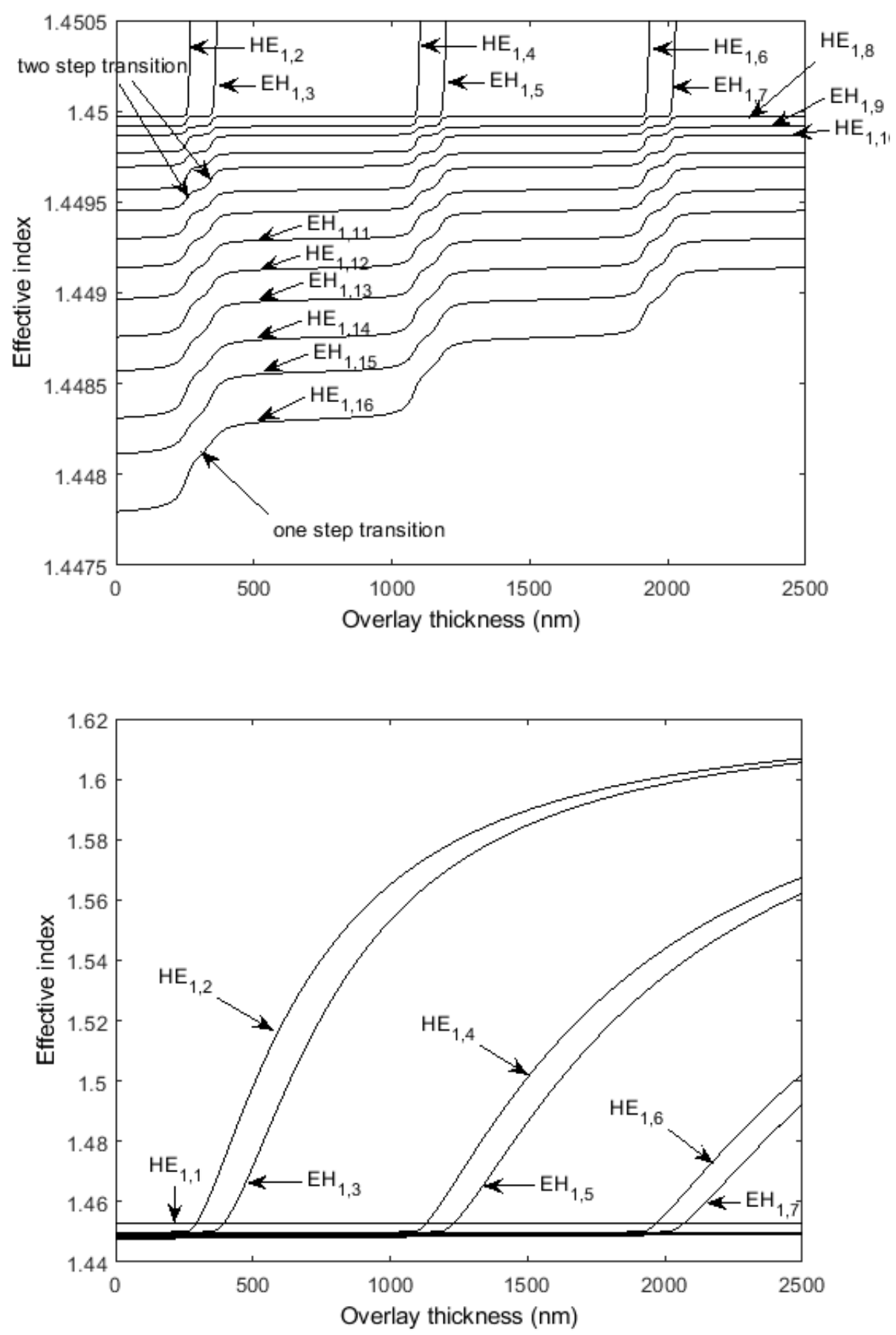
$n^{2} \operatorname{Im}\left(E_{r}\right)(V / m) \quad n^{2} \operatorname{Im}\left(E_{r}\right)(V / m) \quad n^{2} \operatorname{Im}\left(E_{r}\right)(V / m) \quad n^{2} \operatorname{Im}\left(E_{r}\right)(V / m)$
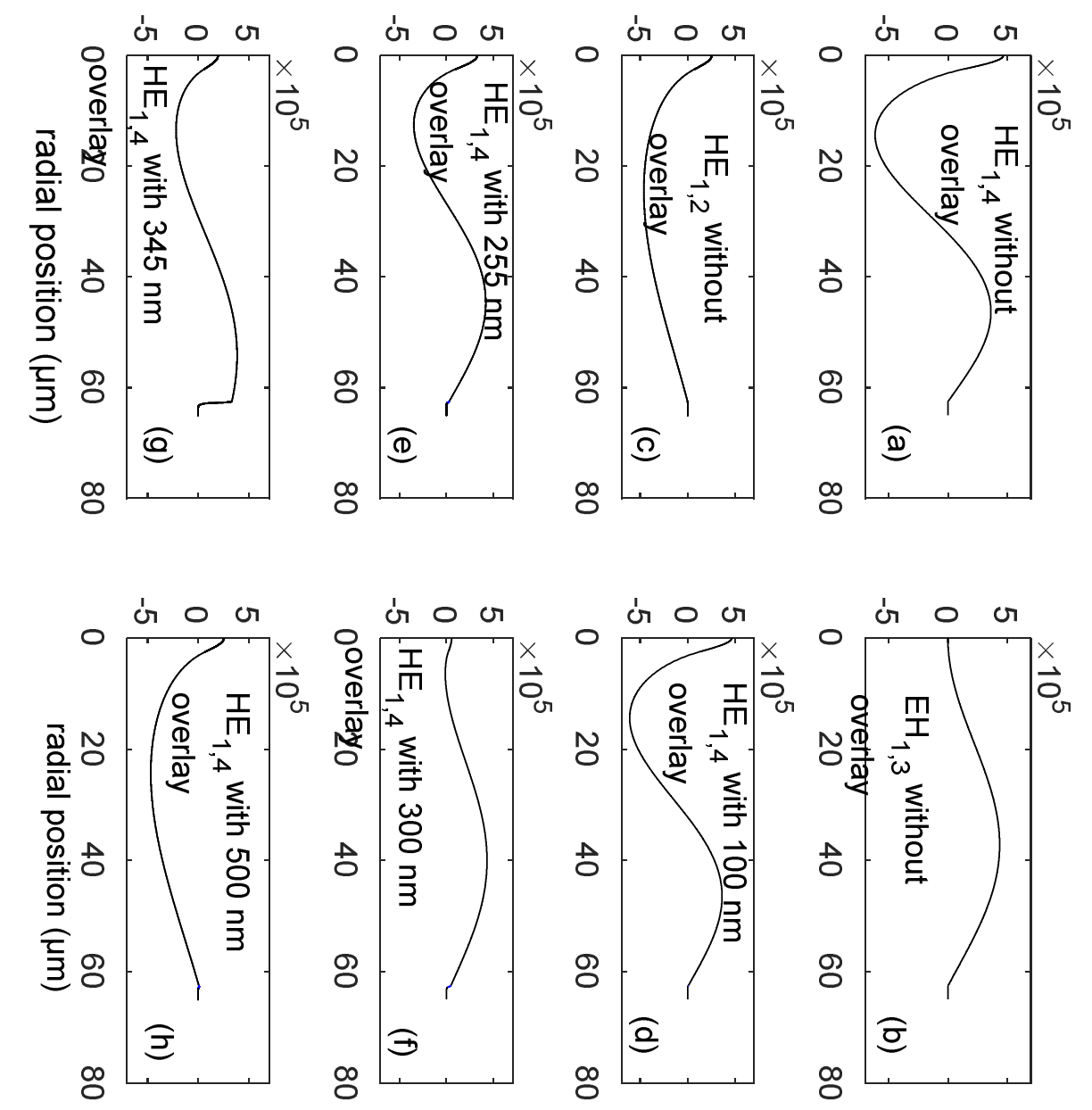
Fig. 4
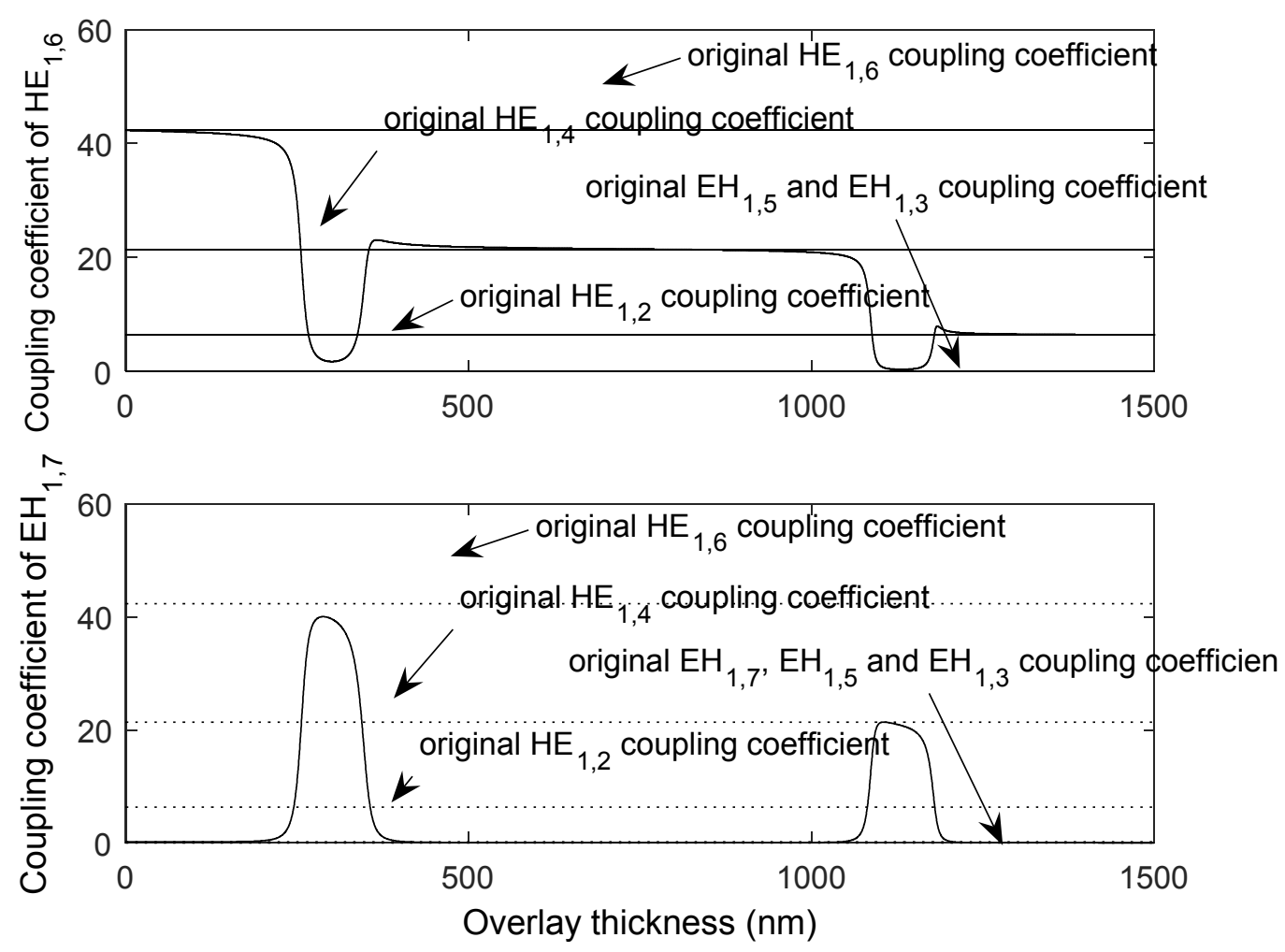
Fig. 5
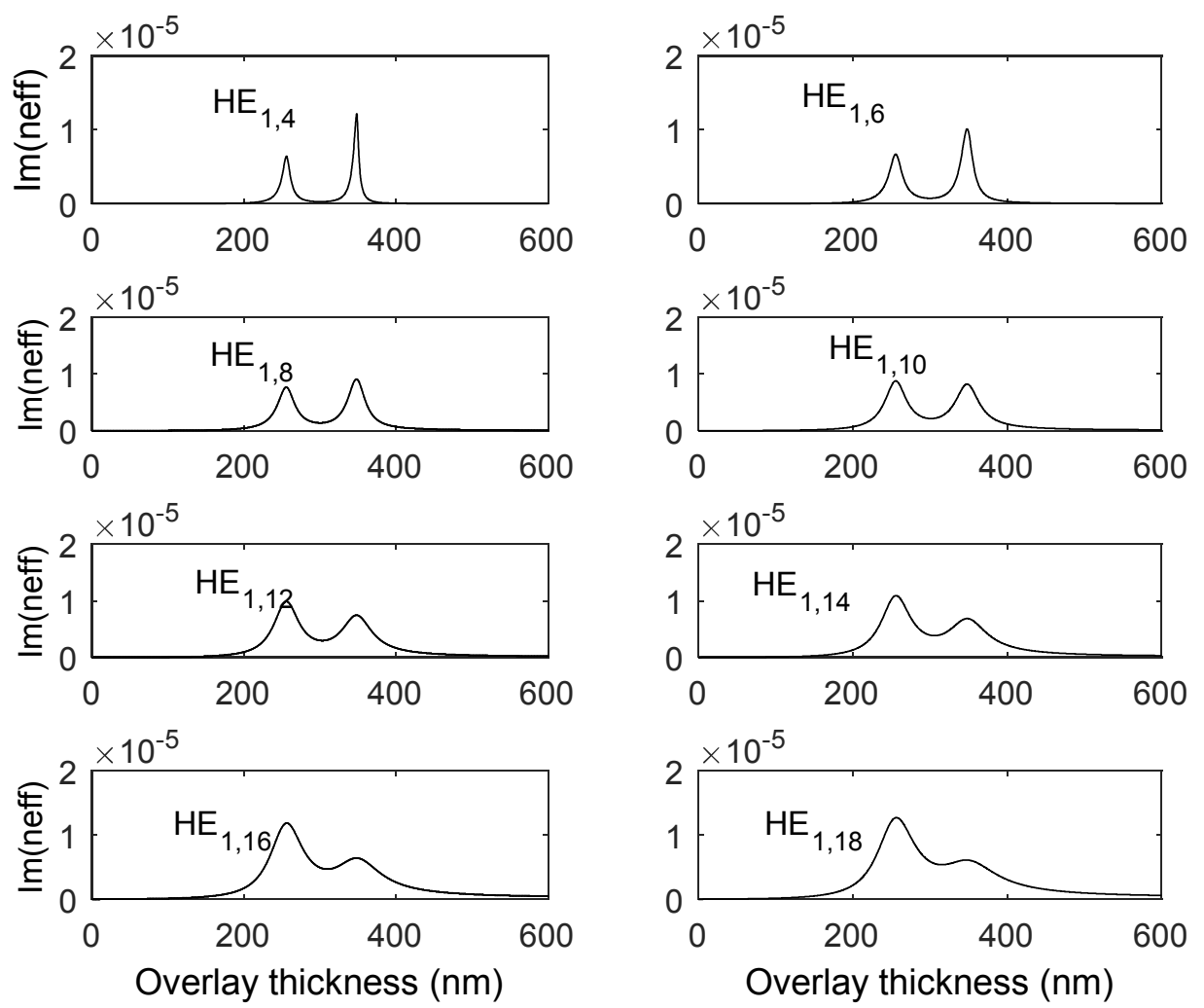
Fig. 6

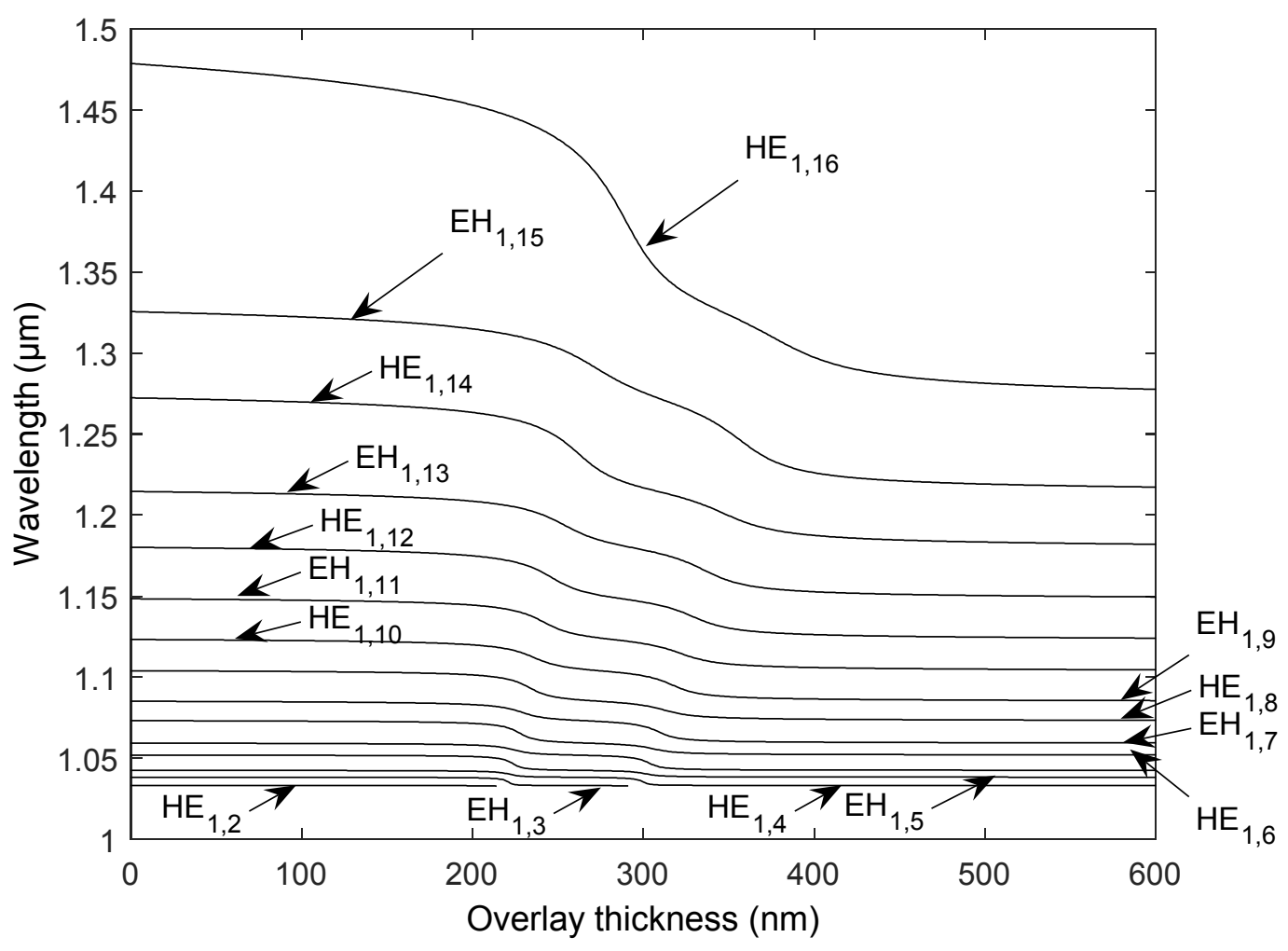


Fig. 7

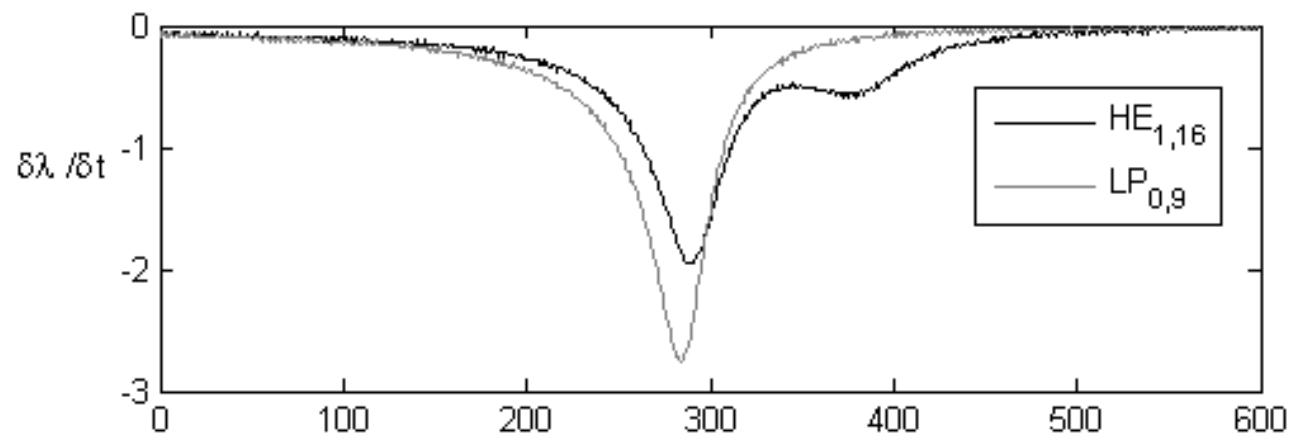

(a)

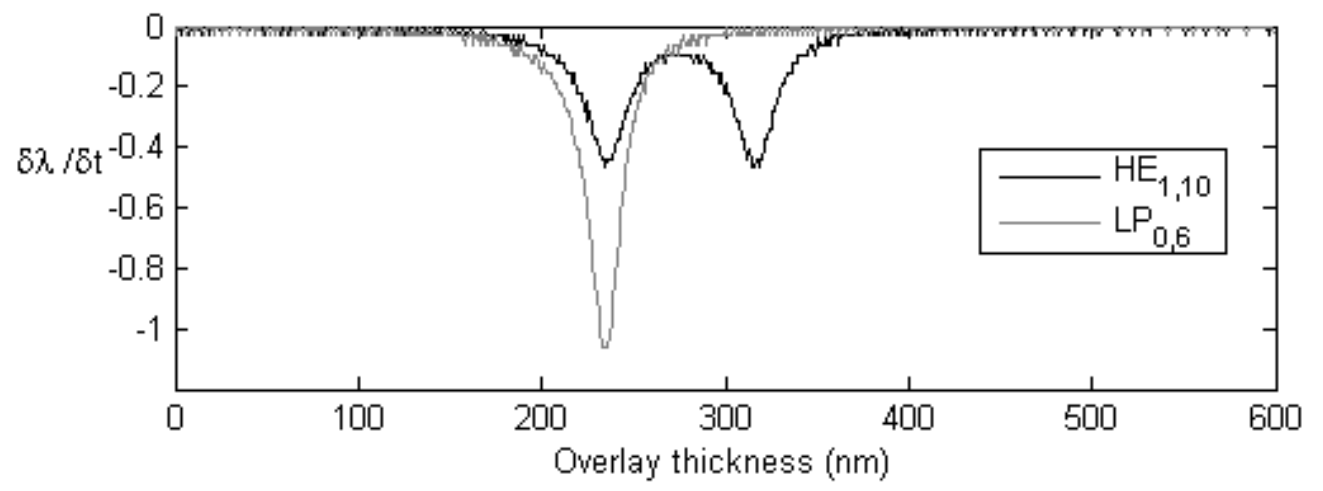

(b) 
Fig. 8

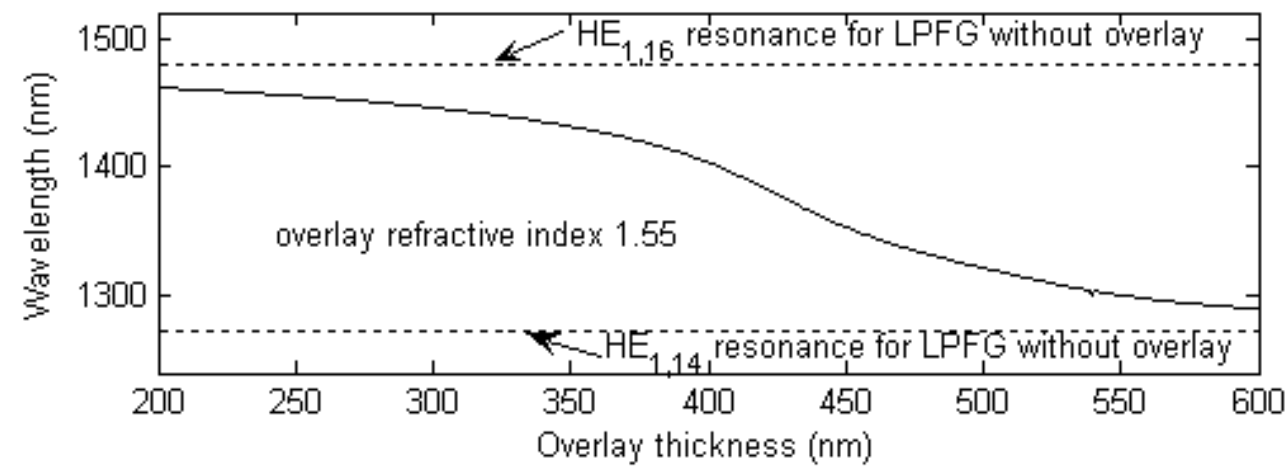

(a)

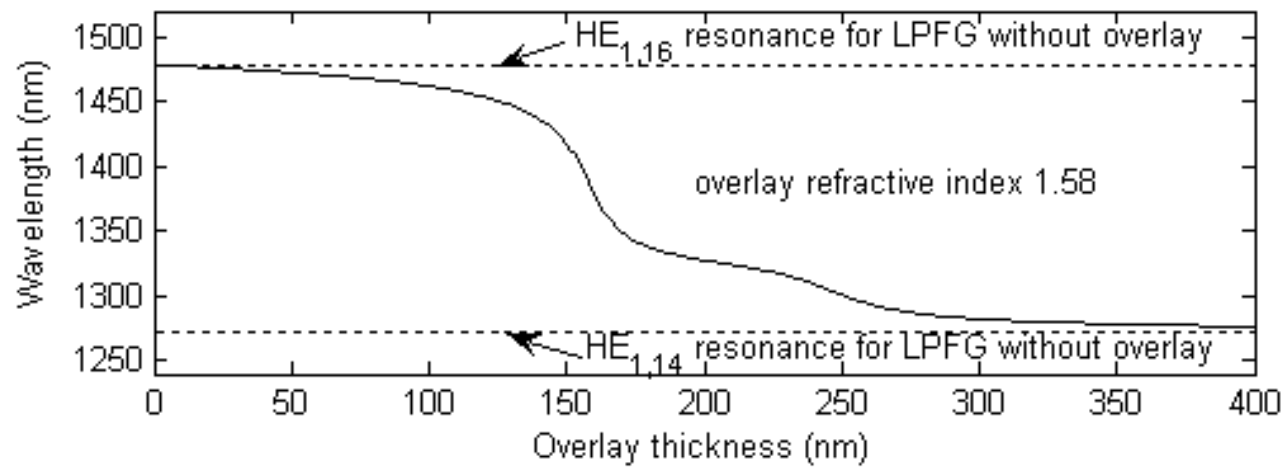

(b) 
Fig. 9

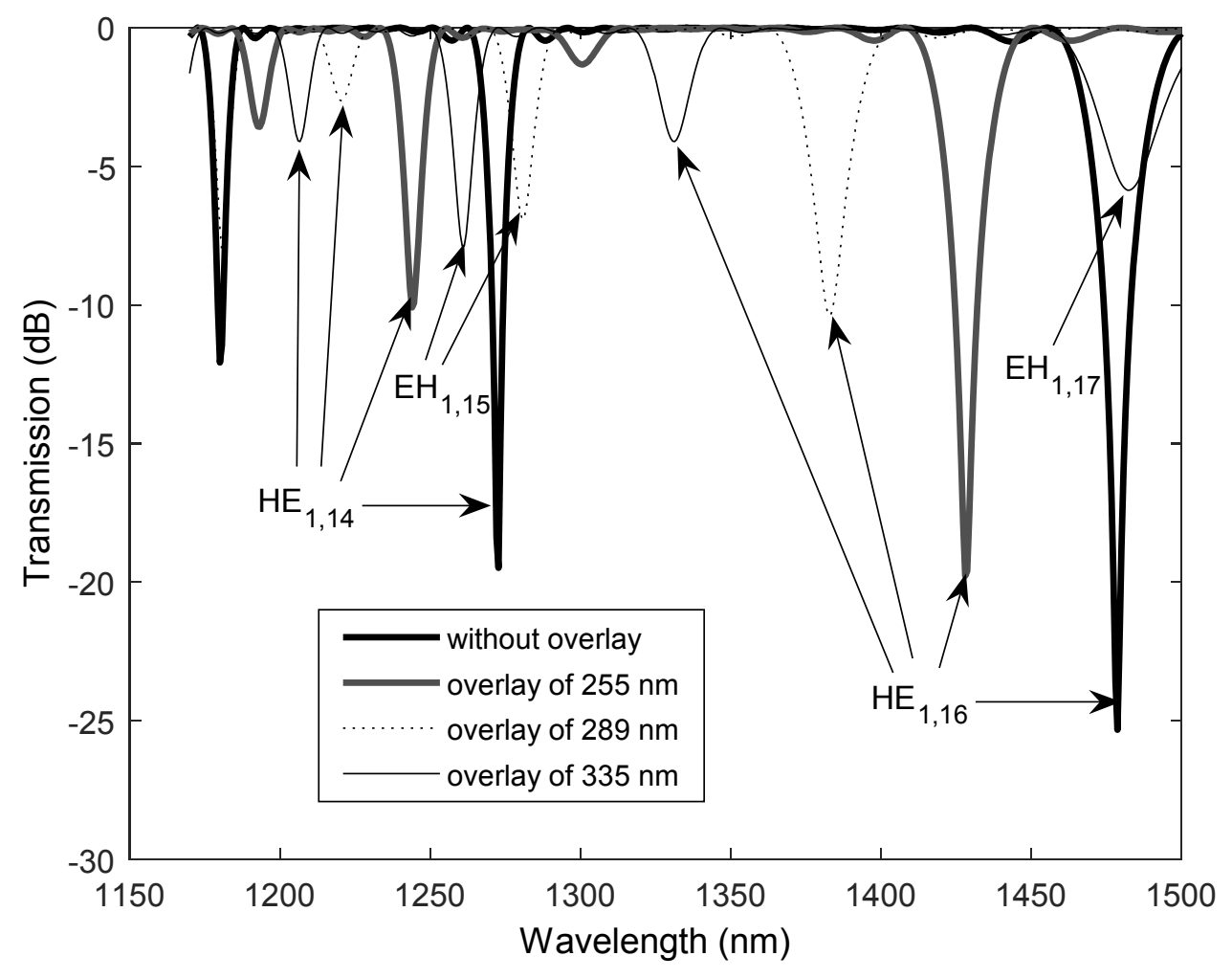


Fig. 10
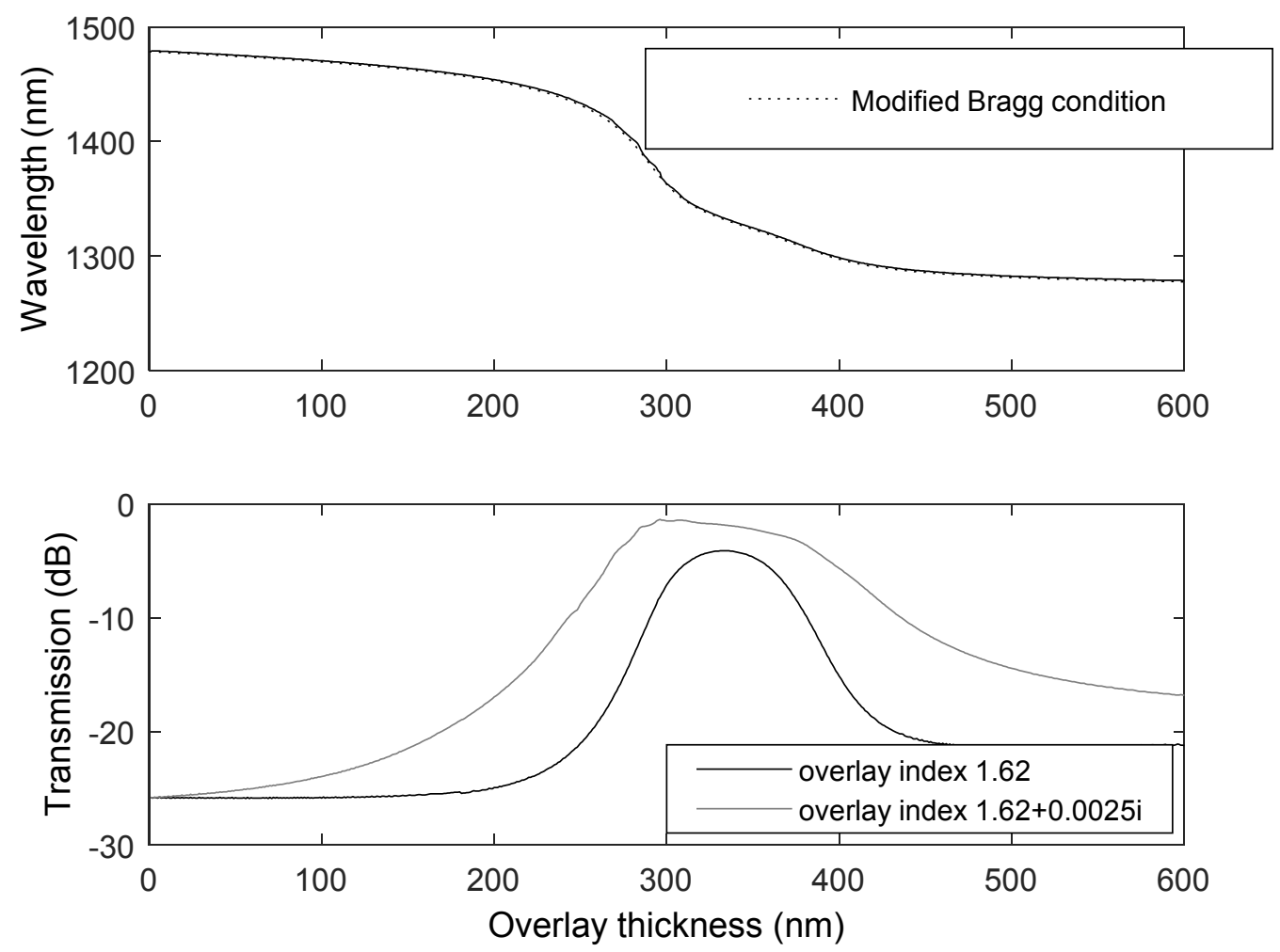
Fig. 11

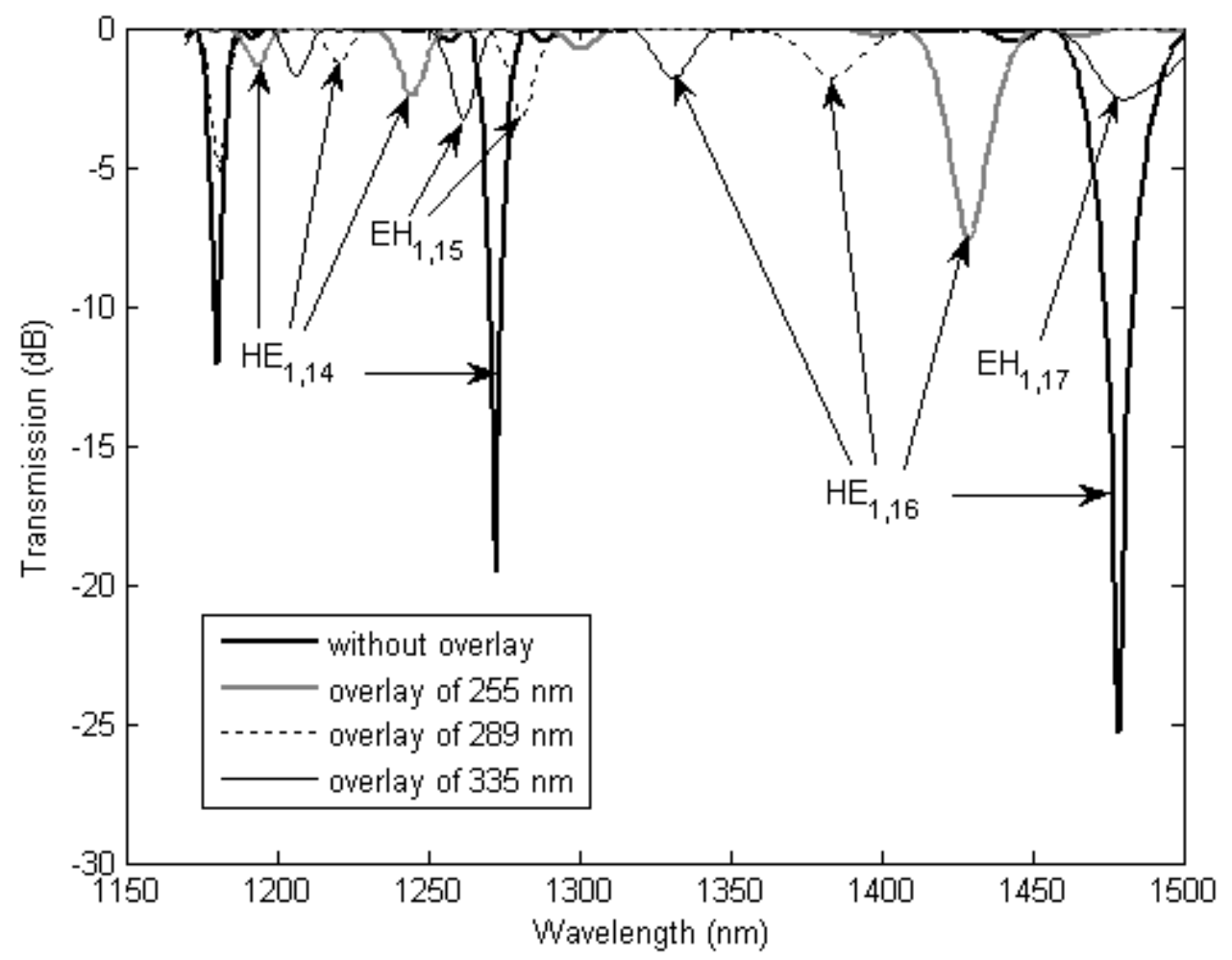

